\title{
Supraventricular tachycardia associated with phentermine use
}

\author{
Pakpoom Tantrachoti MD, Saranapoom Klomjit MD, \\ Supannee Rassameehiran MD, Scott W Shurmur MD
}

\begin{abstract}
Phentermine, a very popular diet pill, is reportedly associated with tachycardia but rarely with other cardiac arrhythmias. We report a 36-year-old woman with no significant past medical history who developed supraventricular tachycardia after taking phentermine for four months. The supraventricular tachycardia has not recurred after the patient stopped taking the medication. With growing prevalence of obesity, clinicians should be aware of the potential serious side effects of phentermine and people with high cardiovascular risk should avoid using this medication.
\end{abstract}

Key words: phentermine, supraventricular tachycardia, obesity

\section{INTRODUCTION}

Obesity has become a major health problem with increasing prevalence, associated comorbidities, and cardiovascular mortality. Pharmacotherapy is a popular option for the management of obesity, and phentermine is one of the most used medications. Side effects of phentermine include insomnia, dry mouth, dizziness, hypertension, and tachycardia. Other cardiac arrhythmias have been rarely reported. ${ }^{2}$ To raise clinicians' awareness, we report the case of a previously healthy young woman who developed supraventricular tachycardia (SVT) after the use of phentermine.

Corresponding author: Pakpoom Tantrachoti MD Contact Information: p.tantrochoti@ttuhsc.edu DOI: 10.12746/swrccc2016.0415.206

\section{CASE}

A 36-year-old woman with no significant past medical history presented with palpitations for one day. She started to notice abnormal heart beating while driving, felt dizzy, and vomited twice. She denied chest pain, shortness of breath, cough, and fever. The patient visited her primary care doctor who sent her to the emergency care center after it was found that she had marked tachycardia. Her initial vital signs showed HR 194 bpm, BP 114/80 mmHg, T 97.8 F, RR 16/ min, and $97 \%$ oxygen saturation on room air. Her BMI was $34.36 \mathrm{~kg} / \mathrm{m}^{2}$. An ECG showed supraventricular tachycardia (SVT) rate 190 bpm (Figure 1) consistent with atrioventricular nodal reentrant tachycardia (AVNRT). However, atrial tachycardia and/or atrioventricular reentrant tachycardia (AVRT) could not be ruled out. Intravenous metoprolol $(5 \mathrm{mg})$ was administered. Follow-up ECG revealed normal sinus rhythm at a rate of $93 \mathrm{bpm}$ with no significant ST-T changes (Figure 2). Laboratory tests showed normal electrolytes (Na $138 \mathrm{mmol} / \mathrm{L}, \mathrm{K} 3.8 \mathrm{mmol} / \mathrm{L}, \mathrm{Cl} 104 \mathrm{mmol} / \mathrm{L}$, 
$\mathrm{HCO}_{3} 20 \mathrm{mmol} / \mathrm{L}, \mathrm{BUN} 24 \mathrm{mg} / \mathrm{dL}, \mathrm{Cr} 0.7 \mathrm{mg} / \mathrm{dL}, \mathrm{Ca}$ $9.5 \mathrm{mg} / \mathrm{dL})$. However, cardiac enzymes were slightly elevated (CK 215 unit/L, CKMB 8.3 ng/ml, Troponin $\mathrm{T} 0.12 \mathrm{ng} / \mathrm{ml}$ ). The patient was initially treated with an ACS protocol with aspirin, clopidogrel, atorvastatin, enoxaparin, carvedilol, and lisinopril. Her urine drug screen was negative, and her thyroid function tests were within normal values. On further inquiry, the patient stated that she had been taking phentermine $(37.5 \mathrm{mg}$ ) one tab daily for four months and she had lost about 15 pounds since she started taking it. The patient was admitted for overnight observation in a cardiac intensive care unit. Serial troponin $\mathrm{T}$ levels at six and 12 hours intervals were 0.14 twice. Echocardiography demonstrated normal ejection fraction with no regional wall motion abnormality. A Lexiscan stress test revealed no evidence of stress-induced ischemia. The patient's heart rhythm converted back to normal sinus rhythm shortly after admission. She did not develop any SVT during hospitalization, and she was discharged with oral carvedilol. The patient came to follow up in our clinic one month later. She was in sinus rhythm, and she had not had any palpitation, dizziness, or chest pain since being discharged.

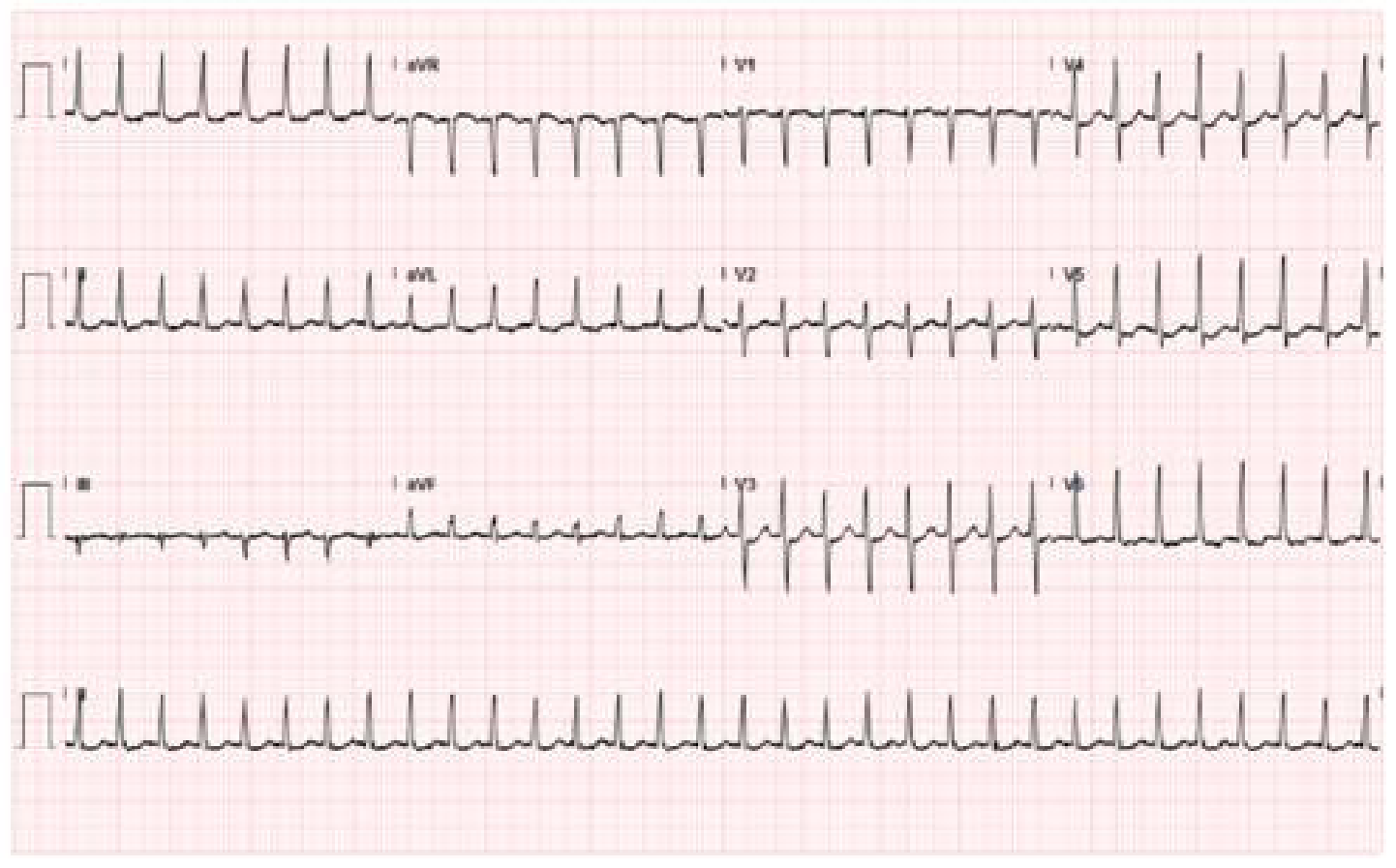

Figure 1: ECG showed supraventricular tachycardia with the rate of $190 \mathrm{bpm}$ consistent with atrioventricular nodal reentrant tachycardia (AVNRT), but atrial tachycardia and/or AVRT could not be ruled out. 


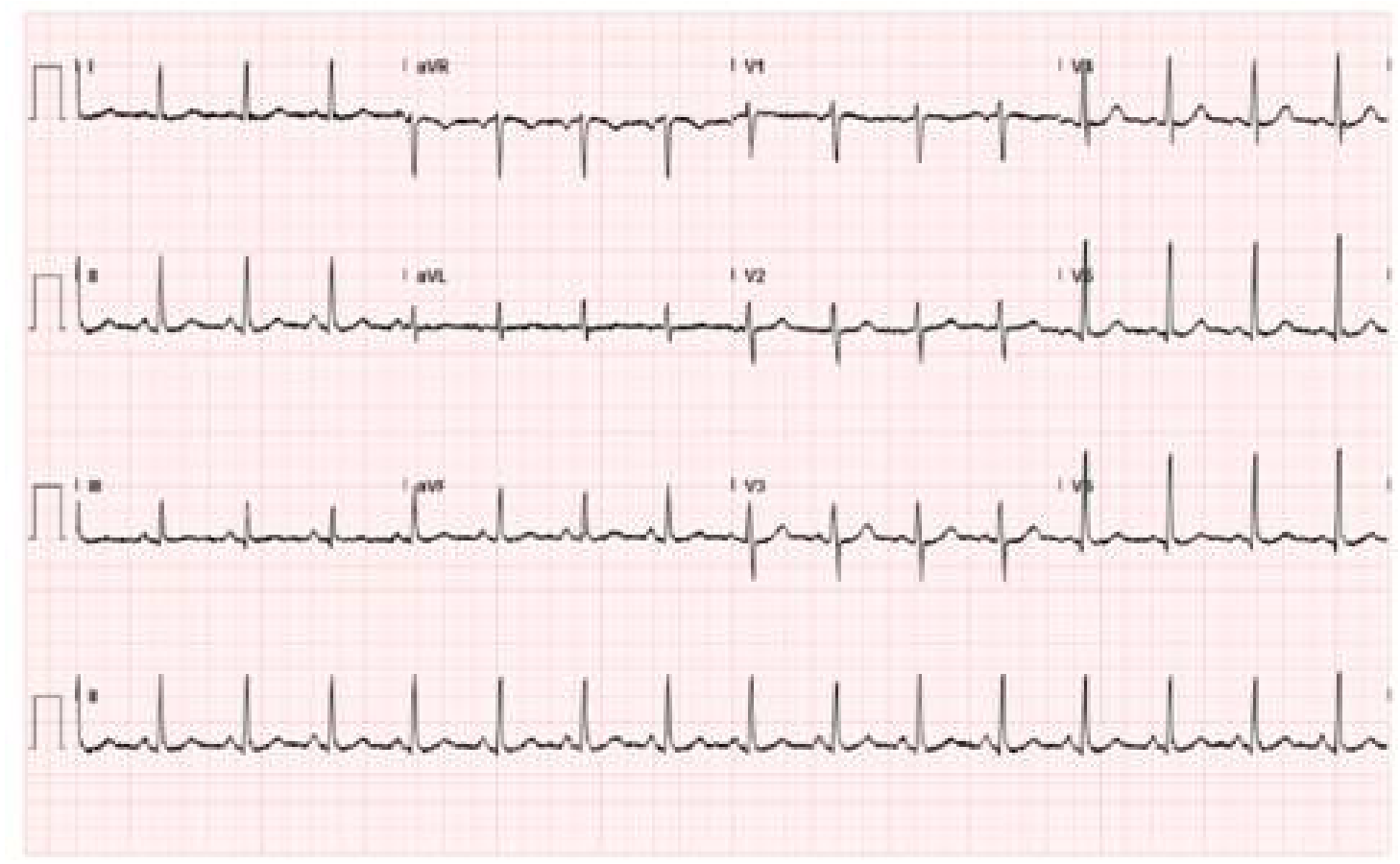

Figure 2: ECG of the same patient after resolution of SVT showed a normal sinus rhythm.

\section{Discussion}

From 1998 to 2008, the prevalence of obesity has doubled in every region in the world. ${ }^{3}$ It affects over ten percent of adults worldwide (11\% of men and $15 \%$ of women) and $35.7 \%$ of the adult U.S. population. ${ }^{4,5}$ Treatment of obesity includes dietary modification, exercise, cognitive behavioral therapy, pharmacotherapy, and surgery. ${ }^{6}$ Successful treatment requires self-determination as the first step. However, some people seek an easy and quick option to lose weight, including the use of weight-reducing products. They disregard or sometimes are unaware of potential harm that may occur after using these remedies.

Phentermine has been approved for shortterm (three months) use for weight reduction by the U.S. Food and Drug Administration (FDA) since 1959, and it is the most frequently prescribed weight-reducing medication in the United States. ${ }^{1}$ Phentermine, a sympathetic amine, is an adrenergic reuptake inhibitor, and it works by augmenting the adrenergic signal- ing in the central nervous system and peripheral tissue. ${ }^{7}$ It stimulates the hypothalamus via $\beta$-adrenergic receptors to suppress appetite and act on the sympathetic nervous system to increase resting energy expenditure. This anorectic agent became well-known in the mid-1990s when "Fen-Phen" (fenfluroxaminephentermine) produced significantly more weight loss than either of them alone resulting in widespread use. The combination was taken off the market in 1997 after it was found that fenfluroxamine or the closely related desfluroxamine was associated with increased incidence of cardiac valvular abnormalities. Phentermine, however, is still available as it has no known valvular heart effect. ${ }^{6.7}$ Later in 2012, phentermine/ topiramate was approved by the FDA and became the first combination drug for long-term management of obesity. ${ }^{1}$ Data from meta-analysis showed that patients taking phentermine lost $3.6 \mathrm{~kg}(\mathrm{Cl}, 0.6-6.0 \mathrm{~kg})$ on average compared with placebo.

Side effects of phentermine include tachycar- 
dia, hypertension, dry mouth, anxiety, insomnia and constipation. ' Kim et al did a post marketing surveillance study on 837 obese patients on phentermine, and this revealed a $30 \%$ incidence rate of adverse effects. The three most common side effects were insomnia $(11.4 \%)$, dry mouth $(5.9 \%)$, and dizziness $(2.7 \%)$. Tachycardia occurred in only $0.4 \%$ of the participants in this study, and no SVT or ventricular arrhythmias have been reported. ${ }^{6}$ Although monotherapy with phentermine has been approved by the FDA for only three months, longer use of the medication is not uncommon in clinical practice to achieve and maintain weight reduction.

There are case reports of ischemic stroke, myocardial infarction, ventricular arrhythmia, and cardiac arrest associated with phentermine use. Three cases of ventricular arrhythmia associated with phentermine use have been reported in the literature. The first one was a 23-year-old previously healthy woman who developed prolonged QT intervals and polymorphic VT after taking a weight-lowering regimen containing phentermine and chlorpheniramine. The patient's condition improved after discontinuation of the medication. ${ }^{11}$ The second case was a 48-yearold woman with no significant medical history who had an episode of ventricular tachycardia followed by ventricular fibrillation arrest requiring defibrillation and intubation. The patient was not taking any medications except phentermine for weight loss. She was subsequently found to have myocardial infarction and was discharged after receiving an implantable cardiac defibrillator. ${ }^{12}$ The third case was a 70-year-old type 2 diabetic woman who presented with documented ventricular fibrillation after starting phentermine therapy for three days. The patient had an episode of ventricular fibrillation arrest during hospitalization and emergent cardiac catheterization revealed right coronary artery vasospasm. ${ }^{2}$ Phentermine should be used with caution in patients with high risk of cardiovascular complications, and patients need to be extremely cautious if they are taking other medications with cardiac side effects, such as antihistamines which can prolong QT intervals together with phentermine.

Other causes of SVT in the differential diag- nosis include structural heart disease, thyrotoxicosis, and drugs. In our case, the patient was young and healthy with no prior history of heart disease. Her thyroid function test was normal; a urine drug screen was negative. Echocardiography showed no evidence of structural abnormality. The only medication that the patient was taking prior to hospitalization was phentermine. In susceptible individuals, supraventricular tachycardia may be precipitated by strong emotion, alcohol, or caffeine..$^{13}$ Our patient did not have a history of emotional stress at the onset of cardiac arrhythmia, and denied alcohol and caffeine ingestion. Therefore, we propose that the supraventricular tachycardia reported in our patient was associated with phentermine use.

In conclusion, we report a case of supraventricular tachycardia in a healthy patient taking a popular weight-reducing drug, phentermine. This medication can result in mild adverse effects; patients and clinicians should remember its potential serious side effects, including cardiac arrhythmias. Patients with high cardiovascular risks, including concurrent use of medications with cardiac side effects and underlying structural heart diseases, need to be extremely cautious before starting this medication.

Author Affiliation: Pakpoom Tantrachoti, Saranapoom Klomjit, and Supannee Rassameehiran are residents in Internal Medicine at Texas Tech University Health Sciences Center in Lubbock, TX. Scott W Shurmur is a chief of Cardiology in the Department of Internal Medicine at TTUHSC in Lubbock, TX.

Received: $05 / 16 / 2016$

Accepted: 07/11/2016

Reviewers: Anurag Singh MD

Published electronically: 07/15/2016

Conflict of Interest Disclosures: none 


\section{REFERENCES}

1. Shukla AP, Buniak WI, Aronne LJ. Treatment of obesity in 2015. J Cardiopulm Rehabil Prev 2015;35(2):81-92.

2. Tobbia P, Norris LA, Klima LD. Ventricular fibrillation coinciding with phentermine initiation. BMJ Case Rep 2012;2012.

3. Ogden CL, Carroll MD, Kit BK, Flegal KM. Prevalence of obesity and trends in body mass index among US children and adolescents, 1999-2010. JAMA 2012;307(5):483-90.

4. WHO. Global status report on noncommunicable diseases 2014. 2015:78-9. Available from: http://www.who.int/nmh/publications/ncd-status-report-2014/en/

5. Ogden CL, Carroll MD, Kit BK, Flegal KM. Prevalence of childhood and adult obesity in the United States, 2011-2012. JAMA 2014;311(8):806-14.

6. Kim HO, Lee JA, Suh HW, Kim YS, Kim BS, Ahn ES, et al. Postmarketing surveillance study of the efficacy and safety of phentermine in patients with obesity. Korean J Fam Med 2013;34(5):298-306.

7. Kaplan LM. Pharmacological therapies for obesity. Gastroenterol Clin North Am 2005;34(1):91-104.

8. Li Z, Maglione M, Tu W, Mojica W, Arterburn D, Shugarman LR, et al. Meta-analysis: pharmacologic treatment of obesity. Ann Intern Med 2005;142(7):532-46.

9. Kokkinos J, Levine SR. Possible association of ischemic stroke with phentermine. Stroke 1993;24(2):310-3.

10. Azarisman SM, Magdi YA, Noorfaizan S, Oteh M. Myocardial infarction induced by appetite suppressants in Malaysia. $N$ Engl J Med 2007;357(18):1873-4.

11. Hung YM, Chang JC. Weight-reducing regimen associated with polymorphic ventricular tachycardia. Am J Emerg Med 2006;24(6):714-6.

12. Makaryus JN, Makaryus AN. Cardiac arrest in the setting of diet pill consumption. Am J Emerg Med 2008;26(6):732 e1-3.

13. Delacretaz E. Clinical practice. Supraventricular tachycardia. N Engl J Med 2006;354(10):1039-51. 